\title{
Internet of Things: A Beginners' Précis and Future Scope
}

\author{
Amit Joshi*, Gurpreet Singh and Gagandeep Singh \\ Lovely Professional University, Phagwara - 144411, Punjab, India; \\ amitjoshi956@gmail.com,gurpreet.17671@lpu.co.in, gagandeep.17672@lpu.co.in
}

\begin{abstract}
Background/Objectives: The chief objective of this paper is to enlighten the society, especially youth and beginners, about the Internet of Things paradigm and its applications in various scenarios. Methods/Statistical analysis: The methodology adapted to study the objective and the various incorporating technologies is through extensive literature survey. Several journals, research papers, handbooks and published articles had been referred to during the course of elaboration of this paper. The Internet has been a valuable asset for the study of the emerging terminologies. Findings: The major findings from this study are mentioned - Applications of IoT: The Internet of Things finds its applications in various scenarios ranging from individual level, like as house security, utilities, and personalization to global level, like automated machines and systems, traffic monitoring system, smart rescue facilitation systems. Advantages and disadvantages of employment of IoT: The employment of IoT features the advantages like ubiquitous network, connected computing, reduction of efforts, and economy of energy and currency, whereas the disadvantages are privacy issues, compatibility issues, security threats and complexity. Challenges and Future scopes: The developments in IoT paradigm still face challenges like security of data, data management, and network architecture. The existence of these challenges breed the future scopes in the development of IoT based systems, like increased security, efficient data management, and enhanced architectures. The exposure to these findings shall spark new ideas towards overcoming the challenges faced in development and support the development of new IoT based systems. Application/Improvements: The purpose of this paper is also to cater these findings for future references for knowledge addition, and to give insights towards development of smart systems for a sustainable environment.
\end{abstract}

Keywords: Internet, Network, Sensors, Smart Systems, Things

\section{Introduction}

"Necessity is the mother of invention" - Technology has been advancing $\underline{\underline{1}}$ ever since the intelligence of the human race has achieved new levels. The evolution of mankind has witnessed a thirst of knowledge - curiosity. The world around us is getting smarter now. Providing more sophisticated information and handy features has been the matter of concern for the modern era. Each of the past three centuries has witnessed a novel technology. Information gathering, processing, and distribution were the key technologies $\underline{2}$ during the 20 th century.

The advancement in the exchange of information gave rise to the networking technology ${ }^{\underline{3}}$. Human efforts reduced to a greater extent. The revolutionary change was the replacement of post cards and mails with electronic mail services (known as e-mail), and a cosmopolitan employment of the GPS technology for tracking and identification of location. The installation of telephone networks, the invention of radio and television systems, communication satellites, and the Internet, indeed, are the other developments of great significance, witnessed by the $20^{\text {th }}$ century era ${ }^{4}$.

Now, consider a scenario where Stacy, Editor at Future-Today magazine, is driving home from work, on a cold day. After a little while she finds herself stuck in a traffic jam, and has an intense urge for a warm cup of coffee, as soon as she gets home. Stuck amidst the traf-

*Author for correspondence 
fic, she calls home asking her mother to prepare a warm cup of coffee. And, by the time she has parked her car in the garage and steps into the home, she finds her coffee ready to be slurped... But, what would happen if she called her mother and found that her mother had went shopping with her mates. Who would prepare her coffee now? She now wishes if there was a way that she could tell the coffee machine at home to prepare her coffee and by the time she would reach home, she would find the coffee ready. Sounds funny, right? But, this is certainly possible! To refer such technology, the term Internet of Things $\boldsymbol{s}^{1.5}$, is the call.

The Internet of Things (abbreviated as IoT) is, actually, a network of physical objects or things, like devices or gadgets, automobiles, and even the big buildings that are embedded with electronic devices, guided and managed by an operating software, sensors to collect information about surroundings, and a network connectivity that enables these objects to collect and exchange data, and perform a set of activities or a task.

\section{Conceptualization}

Since the creation of first electronic telegraph in the $19^{\text {th }}$ century to the creation of first web page in 1991 until the year 1999, significant discoveries and inventions have been made in the era of computing. It was the year 1999, when the assistant Brand Manager of Procter and Gamble, Kevin Ashton, coined the term, "Internet of Things". The idea behind, emerged from the very technology of RFID that Kevin had been working with, at P\&G.

An object embedded with an RFID tag enables itself to be sensed and identified by an RFID reader ${ }^{6}$. Thus, the technology can be engaged to identify and group, similar type of objects, and hence this technology became very popular and earned wide acceptations in supply chain industry. Additionally, the fact that the cheaper prices and the range of detection of an object by the use of the RFID technology is broader as compared to the Bar-code and others, verified it as more preferable, and has found its applications in various business sectors like inventory management, logistics and transportation, electronic surveillance systems and many more ${ }^{7}$. However, the technology has its roots back since the World War II, in the form of radar systems. Consequently, it can be said that the trait of the technology - to identify an object, and a close acquaintance with the RFID systems at $P \& G$ would have sparked the idea of a network of things, which can be detected, in Kevin's mind.

An article published in June, 2009, “That 'Internet of Things' Thing”르, clarified Kevin's vision and the idea behind the Internet of Things. It voiced -

"The problem is, people have limited time, attention and accuracy-all of which means they are not very good at capturing data about things in the real world. And that's a big deal. We're physical, and so is our environment. Our economy, society and survival aren't based on ideas or information-they're based on things... If we had computers that knew everything there was to know about things - using data they gathered without any help from us - we would be able to track and count everything, and greatly reduce waste, loss and cost."

The inference deduced from Kevin's words reveal that he envisioned an environment with physical things that could collect data implicitly rather than ceaseless human interactions to provide every minute detail; as he stated that 'people have limited time, attention and accuracy'. He aspired of computer systems that could automatically and intelligently interpret data and produce services or perform certain tasks guided by minimum number of instructions that would solve our daily life problems, efficiently and economically, and would lead to the reduction of waste, loss and cost.

\section{Components of loT}

In the coffee machine scenario illustrated in the beginning section, the user would notify the coffee machine by a message or a voice mail or any alternate means, so that by the time she had reached home, the coffee was ready. Now, to implement such a scenario in real life, the backend process must be broken down into a set of distinct events that would occur during the whole process, as followed -

1. Generation of request by the user.

2 .Propagation of request through a channel, to the other end.

3. Interpretation of request and generation of response by the machine (or things).

4. Propagation of the generated response back to the user.

5. Notification to the user.

As the things are said to exchange different kind of information they must possess the ability to sense and to 
be detected as well as must be connected to a channel or a medium to exchange the information with the user. Also, the things must be intelligent enough to interpret and process the information effectively, in real time, and generate a response or perform the task, with high efficiency ${ }^{1}$.

So, after analyzing the set of events that occurred in the scenario, the main components, whose association will constitute to a system based on the Internet of Things, are -

1. The 'things'

2. Sensors

3. Transmitter and receiver devices

4. Communication channel

\subsection{The 'things'}

As stated in the words of the father of IoT, Kevin Ashton,

"The problem is, people have limited time, attention and accuracy - all of which means they are not very good at capturing data about things in the real world." 8

He mentioned that people's time is limited and to capture details and explicitly provide them to the different things to accomplish a task is a significant problem of the real world, because it would consume time and the process would not be economical. In simple words, in the earlier scenario, if Stacy's machine did not have the capability to take instructions and interpret them, then she might not get her cup of coffee ready by the time she would reach home, rather she would have to drive all the way back home and prepare the coffee herself, which is very less desirable by an individual after a hectic day at work.

Kevin's words reveal that in order to timely and quickly, economically, and the most prominent, smartly, accomplish our daily tasks, the things around us should be capable to gather information automatically, without our help, and plan the future tasks accordingly ${ }^{5}$. For an instance, suppose if your shower knew what music you like to listen to while showering yourself; if your refrigerator could estimate and prepare a list of the grocery items of requirement, the daily efforts of an individual could be reduced to an extent and the routine could be managed efficiently.

The things can be anything - a washing machine or a microwave oven, a vault or a wristwatch, a car or a public transportation bus, or even a house too. Any physical object that can be connected to a network to exchange information can be regarded as the things in Internet of Things.

\subsection{Sensors}

Like the human eyes perceive the objects in the real world, the ears listen to the sound present in the environment, and the nose smells and make distinction between two different odors, similarly, the things in an IoT infrastructure shall require its sensory parts that enable the system or the things to sense the information from its surroundings, and to be sensed or detected. Sensors come into role here. The sensors are responsible to collect the different type of information ${ }^{11}$, required to perform a set of activities to accomplish the desired task. For example, Mark, a health-conscious person, goes for a morning walk every day. When he returns home from the walk, he wants the door to be unlocked and his glass of milk that he kept in the microwave oven before leaving, to be warm. A sensor present on the window will detect whether the person entering the premise is Mark, and thus the system shall immediately unlock the door and inform the microwave to turn on the heating. Such is the role played by a sensor - to detect the analog signals (physical parameters) from the surroundings and convert them to digital signals that can be measured electrically $\frac{12}{}$. To sense the information present in different forms in the surroundings, following are the broad categories of sensors -

\subsubsection{Temperature Sensor}

As the name suggests, temperature sensor is a component of an electronic circuit that senses the information about the thermal energy of any physical object or a body. Thermal expansion stands out to be the most widely accepted and the simplest phenomenon to measure temperature of a body, such as in a mercury-thermometer $\frac{13}{}$. The electrical sensing of temperature employs various methods like resistive sensing, thermoelectric, semi-conductive $\frac{14}{}$, acoustic, and piezoelectric detection $\frac{15}{}$. A small portion of the object's thermal energy is transmitted to the sensor, which in turn converts this energy into electrical signals and hence sensing of temperature is achieved. Although this data may be imperfect or erroneous due to the pragmatic conditions, it can be made faultless by employing two methods of signal processing: predictive and equilibrium. $\frac{13}{3}$ 
The thermal energy or heat flows between two bodies by the means of conduction, convection and radiation. Based on these modes, temperature sensing is classified into following categories -

1. Contact sensing - requires the physical object or the body being sensed to be in direct physical contact with the sensor, e.g. measuring body temperature by thermometer.

2 .Non-contact sensing - senses the radiant energy emitted by a heat source in the infrared portion of the electromagnetic spectrum.

A temperature sensor can be employed in a case where the data regarding the thermal energy of a body or the surroundings is useful to trigger an action. For example, an RTD (Resistive Temperature Detector) can be used in a passive-open mode, in a smart cook-top appliance, to keep track of the temperature of the gravy being cooked. Hence the information collected can be further utilized to notify the end user when the gravy reaches an alarming temperature and is ready to be served.

\subsubsection{Pressure Sensor}

The pressure sensor is another electronic component that determines the amount of force exerted per unit area of application. The range of accurately measureable pressure extends from fraction of inch of water (low level pressure) to hundreds of thousands of PSI (extreme level pressure $)^{16}$. Two types of pressure measuring techniques have been thus employed in order to measure the varying range of pressure, which are: direct and indirect pressure measurement. The indirect pressure measurement techniques comprise of pressure measurement using electrical sensors. Many pressure measuring principles are used today in electrical pressure measurement. Most of the methods use measurement of displacement or force to determine pressure. Piezoresistive pressure sensors are most widely accepted for the same $\mathrm{e}^{\frac{17}{7}}$.

The application of a pressure sensor can be found in a smart security system. A pressure sensor can be enforced simply on a door or a door-lock to determine the value of pressure tolerated by the door or the door-lock. In case of an extreme or intolerable measured value, signifying a break-in, the security or nearby police department and the guardian can be thus notified of the collected information to take control of the situation and avoid the unbearable circumstances.

\subsubsection{Position and Displacement Sensors}

Position is the location or the coordinates of an object with respect to a defined point of reference, while displacement is defined as the change in position of the object. A position sensor is an electronic component that determines the distance of a physical object from a point of reference, while a displacement sensor involves the detection of movement. Determination of the position and displacement of an object or the distance between two objects can pose many applications in different aspects, such as robotics, traffic control, security systems and many others ${ }^{18}$. These sensory devices are classified into many categories (based on their working principles), of which proximity sensor is widely in use in smart phones, presently. The proximity sensor is more of a threshold sensor that determines a limiting distance to trigger an action $\frac{19}{}$. However; there exist other position sensors too, varying by their modus operandi, classified, basically, into contact and non-contact devices ${ }^{20}$.

To realize the applications of a position or displacement sensor, one can envision an automated environment of automobiles where the auto-pilot motion of two automobiles shall be guided based on the output signals generated by the proximity sensors and the other distance and position sensors embedded on them. The distance between two parallel moving automobiles shall determine one's future action, like overtaking the other or slowing up own speed to let the other one pass by.

\subsubsection{Biosensor}

The biosensor is an integrated sensing device. A bioreceptor and a transducer together constitute a biosensor. The object to be sensed or the target of a biosensor is called an analyte. The bioreceptor is a bio-molecule that is responsible for the recognition of the analyte, while the transducer transforms the event so recognized, into a measurable signal ${ }^{21}$. This signal can be further used to perform various tasks, such as to trigger an activity based on a threshold value of the signal.

The biosensor has found its applications in various aspects in health care (e.g. blood sugar level tests, hemoglobin tests), industrial process management and home automation (face recognition or finger print based security systems) ${ }^{22}$. An IoT based smart door-lock system can be designed incorporating a biosensor which, when a person enters a house's premises or reaches the door and is 
about to knock, will recognize whether the individual is an authentic person or not, and further shall instruct to unlock the door, in the former case.

\subsubsection{Acoustic Sensor}

An acoustic sensor is, fundamentally, a device that is similar to that of the pressure sensor, in terms of working principle (i.e. both operate on mechanical pressure waves), but differs by the approach of collecting information (an audio sensor would not detect a constant or very slow changing pressure). Audio sensors are chiefly termed 'microphones', nonetheless a microphone is often used for infrasonic and ultrasonic sound too ${ }^{23}$. Additionally, a microphone is a transducer that transforms the input sound waves into electrical signals.

An acoustic sensor has a similar structure as that of a pressure sensor, since an acoustic wave is also a mechanical pressure wave. It is constituted by a moving diaphragm and a displacement transducer that converts the diaphragm's movement into an electrical signal. Microphones are further classified into different categories, as mentioned in ${ }^{24}$.

The acoustic sensor plays a vital role in the construct of an IoT based system. One may find an acoustic sensor very useful in a smart voice-commands system, where the system would be solely based upon the recognition of voice commands. A user may ask the smart system to turn ON/OFF the lights or change the ambience of the room, or simply command the AC to stir up the cooling or ask the telly to turn up/down the volume.

Now, having discussed some common varieties of the sensors to effectively construct an IoT based system (however, there are many other classifications of sensors like flow sensor, velocity sensor, level sensor) ${ }^{25,26}$, it is necessary to make the choice of the appropriate sensor. The selection of an aptly fitting sensor is based on the following parameters ${ }^{27}$

1. Accuracy: The precision with which the sensor can process.

2. Environmental condition: The physical conditions of the surroundings.

3. Range: The limit within which the sensor can work.

4. Calibration: Whether the sensor is needed to be calibrated or to be oriented according to some guidelines.

5. Resolution: The capability of the sensor to detect smallest change.

6. Cost: The price of the sensor.
7. Repeatability: The feature of the sensor to operate on a repetitive input.

\subsection{Transmitter and Receiver Devices}

Once the information is collected by the sensor(s), the information is needed to be exchanged in order for the process to move to the next phase and perform the successor event. This exchange of information is carried out by transmitting and receiving devices. A transmitter is generally an electronic circuit that produces or emits the information into its surroundings, with the help of an antenna, while the receiver is an electronic circuit that converts the incoming digital signals to analog signals, such as sound or light that can be perceived by humans. The transmitter and receiver devices operate on radio frequency, generally, for long distance communication. The alternatives could be infrared frequency or Bluetooth

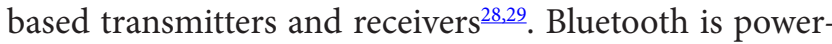
ing different industries with quintessential low cost and flexible development architecture. It is highly expected for Bluetooth technology to see a growth in wireless medical devices, wearables, home automation and retail ${ }^{10}$. The Bluetooth based transmitting and receiving devices can operate within a range of 10 to 100 meters and the infrared transmitters and receivers are scalable within an even smaller range of $1 \mathrm{~mm}$. Hence the radio frequency transmitters and receivers are more preferable.

\subsection{Communication Channel}

There can be different types of communication channels for the propagation of information; they are further broadly classified into the following two categories -

1. Guided media (wired media): These media require a physical medium to transfer the information from one node/end to the other. Some popular guided media are coaxial cables, twisted pair cables and optical fiber cables.

2. Unguided media (wireless media): These media do not require any physical medium for the propagation of the information from one end to the other; rather they operate on different frequency spectrums.

Since, the key feature of the IoT technology is the exchange of data over a large network of things that are generally connected over wireless media. Hence, the communication channel that aptly fits into the scenario is unguided media, and the spectrum of frequency over which the information is propagated is the radio fre- 
quency spectrum. The medium through which the data is transferred complies with IEEE standard $802.11 \mathrm{~b} / \mathrm{g} / \mathrm{n}$.

Further the exchange of data over the channel takes place with the help of a set of protocols and services enforced thusly ${ }^{5}$.

\section{Applications}

With the robust features of the technology, the Internet of Things exhibits a broad field of applicability. The technology can be employed in the field of environmental monitoring, infrastructure management, medical and healthcare systems, media, or home automation. The technology finds different use-cases in these different fields of application. Following are some fascinating applications of the IoT, categorized into three levels according to the scalability -

\subsection{Smart City}

The idea of smart city is not only confined to building attractive infrastructures and fancy technology equipped architectures; rather the main concern is to transform a city into more human friendly and eco friendly, more economic than before (in terms of energy consumption, time, costs and wastes), and more of a place with a sustainable environment. Various measures can be taken into account to transform a city into a smart city ${ }^{5}$. IoT finds applicability in many expanses of the real world to ease many lives as well as making them safer and optimum.

To throw light at the applicability of IoT in developing a smart city, consider a scenario: Imagine Sky Civic, a developing new smart city. The mayor introduces a new development scheme for the city that is composed of -

\section{A Smart Rescue Utility (SMU)}

2. Smart Power Readers (SPRs), and

3. Smart Traffic Optimizers (STOs)

The Smart Rescue Utility (SMU) is a service that will be notified in case of an emergency, like a road accident or a fire. On the detection of an emergency, the $S M U$ will direct a rescue wagon towards the rescue site, containing all the first-aids to relieve the victims at once.

The Smart Power Readers (SPRs) constitute a system that records the monthly power consumption readings from every house of a society and dispenses electronic bills to the users. A Smart Power Reader, on completion of a month, automatically dispatches the records to Sky Civic's electricity control stations where the SPR systems generate the electricity bills which are further dispatched to the users.

The Smart Traffic Optimizers (STOs) system is a traffic management system that analyses the traffic at various locations and manages the traffic by scheduling an optimum waiting time at a traffic light, and also guiding the different vehicles with an optimal path. A Smart Traffic Optimizer is a traffic light that analyses the traffic at the point and schedules an optimum waiting time and notifies the adjacent STOs of the scheduled time in order to brew optimization on a larger scale. Also, it guides the driver of a vehicle by showing on the map, a path with less traffic, an optimal path.

\subsection{Smart Home}

The concept of smart homes is an association of various home automation applications that together constitute a fully automated house. An individual can personalize her/his home and automate it accordingly based on her/ his daily activities ${ }^{1,5}$.

Now, consider a scenario where Mark and Stacy move in together into their new 3BHK flat. One fine day, they are watching the Discovery channel. The show showcases modern technologies for transforming your home into a Future home. Mark and Stacy decide to turn their flat into a fully automated home. They setup a Smart Refrigerator that notifies for all the grocery that is running out, a Smart fire alarm that notifies Mark and Stacy and the fire department as well, in case of a fire emergency at home; the Smart Home Guard detects Mark's face and enables him to enter the house without even Stacy unlocking the door. Mark and Stacy are happy with their new IoT equipped Smart home.

\subsection{Smart Gadgets and Wearables}

Smart gadgets and wearables are the key tools to personalize one's life ${ }^{\frac{1,9}{9}}$. A smart wristband, a smart wearable, helps you track your fitness, activity, logs heart rate, schedule alarms, acts as a pedometer and has many more interesting features. The following scenario will make the idea more clear -

After finally moving into their new home and, after turning their new home into a Smart home, Mark and Stacy, now, also have their new Smart wristband. Mark 
continues his regular morning exercise. He, now, wears his Smart wristband to track the distance he covered and his fitness level. But one day, on his way back home, Mark meets an accident. Fortunately, he is wearing his wristband that detects his lowered heartbeat rate and notifies the nearby hospital to send an ambulance at the point of accident, as well as Stacy about Mark's condition. The ambulance arrives in the right time and saves Mark from the consequences.

There are numerous other applications of the IoT technology that can be contained and demonstrated in this paper ${ }^{1,5}$. One of the future scopes includes the rapidly growing technology in IoT, Raspberry pi. The Raspberry pi is a very small computer (that of the size of a creditcard) and is a very cost effective technology. One can plug it into a computer monitor or TV and use standard keyboard and mouse to operate it. It enables people of any age to explore computing and learn high-level programming languages like Python and Scratch.

\section{Advantages and Disadvantages}

The features advocating the advantages of the Internet of Things are as under -

1. Ubiquitous network: Personal wifi connectivity on every mobile and other portable devices result to a magnificent network around the globe.

2. Connected computing: It enables different devices like mobiles, tablets, televisions, music players, vehicles to keep track of our activities and helps these devices to support to carry our daily tasks.

3. Intelligence at the periphery of a network: According to Jim Gray (10 years ago), "Intelligence is moving to the periphery of the network. Each disk and each sensor will be a competent database machine." which is apparently very certain.

4. Reduction of human efforts on daily life activities and contemporary life issues, to a greater extent.

5. It is an economical technology. Adopting the IoT technology, one can reduce the costs of carrying out different activities and reduce the wastes to great extents.

6. Reinforcement of this technology in health care and medical field can help save many lives.

Although the Internet of Things finds many advantages in the real world, but as it is said, "every coin has two faces", there are several points leading to the disadvantages of the technology too -

1. Privacy issues: With all the data being transmitted, the risk of losing privacy increases. For instance, do one's neighbors or colleagues need to know all the information of the individual?

2. Compatibility issues: Apparently, there are no international standards defined for the tagging and monitoring devices, which may raise compatibility issues.

3. Security threats: Imagine if a hacker alters the medical prescription of an individual, in the database and the medical store ships false medicines to which the user is allergic, the consequences are unimaginable.

4. Complexity: The opportunities of failure rise with the complexity of the system. For instance, if you and your spouse, both receive a notification regarding a need of several grocery items or the expiry of milk. As a result, both may end up buying the listed items and there may be excess stock.

\section{Challenges and Future Scope}

In near future, billions of data spouting devices will be connected to the Internet. The applications of Internet of Things span a wide range of domains, like homes, cities, environment, retails, logistic, industry and health. But with increase in number of devices there are lots of challenges in Io $\mathrm{T}$ that require attention:

1. Security: Since a lot of data is generated and transferred through IoT devices, so security becomes the prime concern especially in wireless networks.

2. Data management: Managing data is extremely difficult task, especially in case of IoT where devices are heterogeneous and they have different way of data representation and semantics. Moreover larger IoT devices lead to explosion of full scale data collection. This will give birth to another problem, i.e. scalability problem. With increase in number of devices, indexing methods should be developed to find an item easily.

3. Network architecture: Finding a flexible, scalable and cross platform architecture is another major goal of IoT. Some architecture are proposed for application specific domains like meter reading, industry specific applications and healthcare but these areas require still more attention. 


\section{Summary}

To draw the summary of the paper, following points can be taken into consideration -

- The Internet of Things, on the very basic level, is a network of smart devices and gadgets (termed things).

- The IoT technology enables the end devices or things to collect and exchange information to solve the real life problems.

- The term Internet of Things was coined by Kevin Ashton in the year 1999 while working with the RFID technology at Procter and Gamble.

- The key components that constitute a fully functional IoT based systems, are -

\section{The things \\ 2. Sensors \\ 3. Transmitter and receiver devices \\ 4. Communication channel}

- The Internet of Things technology finds large number of applications in day-to-day life, varying according to the scalability of problems.

- There are many advantages of the technology, such as ubiquity of the network, enhanced communications and connectivity, and intelligence of devices at the periphery.

- Also, there are facts flashing the demerits of the technology, like privacy and compatibility issues, security threats, and complexity. But if one focuses on the positive aspects of the technology, i.e. focusing on the advantages and overcoming the disadvantages (considering that problems are a way of leaning), great milestones can be achieved.

\section{References}

1. Luigi A, Antonio I, Giacomo M. The Internet of Things: A survey. Elsevier Computer Networks. 2010 Jun; 54(15):2787-97.

2. Andrew T, David W. Introduction. Computer Networks. Pearson Edu. Inc.: USA; 2010. p. 1-3.

3. Andrew T, David W. Uses of computer networks. Computer Networks. Pearson Edu. Inc.: USA. 2010. p. 3-16.

4. History of technology [Internet]. Available from: http:// www.britannica.com/technology/history-of-technology/ The-20th-century\#toc14899.
5. Jayavardhana G, Rajkumar B, Slaven M, Marimuthu P. Internet of Things (IoT): A vision, architectural elements, and future directions. Elsevier Future Generation Computer Systems. 2013 Feb; 29(7):1645-51.

6. Roy W. An Introduction to RFID Technology.IEEE Pervasive Computing. 2006; 5(1):25-9.

7. Badri N, Franklin R, Roy W. RFID Technology and Applications. IEEE Pervasive Computing. 2006; 5(1):22-3.

8. That 'Internet of Things' Thing [Internet]. [cited 2016 Apr 04]. Available from: http://www.rfidjournal.com/articles/ view?4986.

9. Prakash M, Gowshika U, Ravichandran T. A smart device integrated with an android for alerting a person's health condition: Internet of Things. Indian Journal of Science and Technology. 2016 Feb; 9(6):1-2, 5.

10. Bluetooth [Internet]. Available from: https://www.bluetooth.com/what-is-bluetooth-technology/bluetooth.

11. Tom K. Basic sensor technology. Sensor Technology Handbook. Newnes: USA; 2004. p. 1.

12. Jacob F. Sensors, signals, and systems. Handbook of Modern Sensors. Springer: USA; 2010. p. 1-7.

13. Jacob F. Coupling with object. Handbook of Modern Sensors. Springer: USA; 2010. p. 519-20.

14. John F. Sensor types and technologies. Sensor Technology Handbook. Newnes: USA; 2004. p. 531-5.

15. Jacob F. Acoustic temperature sensor; piezoelectric temperature sensors. Handbook of Modern Sensors. Springer: USA; 2010. p. 564-6.

16. Jacob F. Concepts of pressure. Handbook of Modern Sensors. Springer: USA; 2010. p. 375-6.

17. Glenn H. Piezoresistive pressure sensing. Sensor Technology Handbook. Newnes: USA; 2004. p. 411-14.

18. Jacob F. Position, displacement, and level. Handbook of Modern Sensors. Springer: USA; 2010. p. 279-80.

19. Adolfo CM. Proximity sensors. Sensor Technology Newnes: USA. 2004; p. 345-9.

20. Adolfo CM. Types of position sensors. Sensor Technology Handbook. Newnes: USA; 2004. p. 322.

21. Young HL, Raj M. Overview: What is a biosensor. Sensor Technology Handbook. Newnes: USA; 2004. p. 161-3.

22. Young HL, Raj M. Applications of biosensors. Sensor Technology Handbook. Newnes: USA; 2004. p. 164-8.

23. Jacob F. Acoustic sensors. Handbook of Modern Sensors. Springer: USA; 2010. p. 431.

24. John C, Larson D. Common microphone types. Sensor Technology Handbook. Newnes: USA; 2004. p. 482.

25. Jacob F. Handbook of modern sensors. $4^{\text {th }}$ edn. Springer: USA; 2010.

26. Jon SW. Sensor technology handbook. Newnes: USA; 2004.

27. Tom K. Sensor performance characteristics definitions. Sensor Technology Handbook. Newnes: USA; 2004. p. 2-4. 
28. An introduction to infrared technology: applications in the home, classroom, workplace, and beyond [Internet]. Available from: http://trace.wisc.edu/docs/ir_intro/ir_ intro.htm.

29. Bluetooth devices [Internet]. Available from: https://www. bluetooth.com/what-is-bluetooth-technology/bluetoothdevices.

30. Bluetooth. Available from: https://www.bluetooth.com/ what-is-bluetooth-technology/bluetooth.

31. Jayavardhana G, Rajkumar B, Slaven M, Marimuthu P. Internet of Things (IoT): A vision, architectural elements, and future directions. Elsevier Future Generation Computer Systems. 2013 Feb; 29 (7):1649.

32. Jayavardhana G, Rajkumar B, Slaven M, Marimuthu P. Internet of Things (IoT): A vision, architectural elements, and future directions. Elsevier Future Generation Computer Systems. 2013 Feb; 29(7): 1650.

33. Jayavardhana G, Rajkumar B, Slaven M, Marimuthu P. Internet of Things (IoT): A vision, architectural ele- ments, and future directions. Elsevier Future Generation Computer Systems. 2013 Feb; 29 (7):1650-1.

34. 34. Luigi A, Antonio I, Giacomo M. The Internet of Things: A survey. Elsevier Computer Networks. 2010 June; 54 (15):2795.

35. Luigi A, Antonio I, Giacomo M. The Internet of Things: A survey. Elsevier Computer Networks. 2010 June; 54(15): 2796

36. M. Prakash, U. Gowshika, T. Ravichandran. A Smart Device Integrated with an Android for Alerting a Person's Health Condition: Internet of Things. Indian Journal of Science and Technology. 2016 Feb; 9(6): 1-2.

37. Luigi A, Antonio I, Giacomo M. The Internet of Things: A survey. Elsevier Computer Networks. 2010 June; 54 (15):2793-7.

38. Jayavardhana G, Rajkumar B, Slaven M, Marimuthu P. Internet of Things (IoT): A vision, architectural elements, and future directions. Elsevier Future Generation Computer Systems. 2013 Feb; 29 (7):1649-51. 